\title{
A utilização da atividade como ferramenta no processo de intervençáo do terapeuta ocupacional em reabilitaçáo neurológica
}

\author{
Diane Priscila Stoffel ${ }^{\mathrm{a}}$, Renato Nickel ${ }^{\mathrm{b}}$ \\ ${ }^{a}$ Acadêmica do $9^{\circ}$ Período do Curso de Graduação em Terapia Ocupacional, \\ Universidade Federal do Paraná - UFPR, Bolsista de Iniciação Científica, Curitiba, PR, Brasil

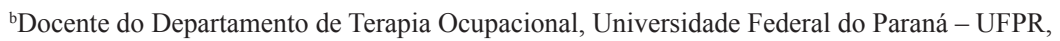 \\ Doutor em Educação, Curitiba, PR, Brasil
}

\begin{abstract}
Resumo: Este artigo é uma reflexão teórica e tem como objetivo discutir a prática do terapeuta ocupacional em reabilitação neurológica, com foco no uso da atividade como ferramenta de intervenção desse profissional. Para isso, propõe-se levantar subsídios entre a estrutura e nomenclatura da Classificação Internacional de Funcionalidade, Incapacidade e Saúde (CIF), os princípios do Modelo de Ocupação Humana e a Abordagem da Aprendizagem Motora para Recuperação do Comportamento Motor. O desempenho de atividades cotidianas, o desenvolvimento de hábitos e a inserção deles na rotina do sujeito com condição neurológica estimulam seus processos de aprendizagem e adaptação. Isso permite que o sujeito adquira e organize novos padrões de desempenho satisfatórios para sua participação. Nesse âmbito, as atividades empregadas pelo terapeuta ocupacional no processo de reabilitação deveriam envolver funções corporais já controladas pelo sujeito, uma vez que a mudança do comportamento motor só ocorre com base na modificação de habilidades já adquiridas. Ressalta-se a necessidade de fundamentação da prática dos terapeutas ocupacionais no escopo teórico da própria profissão com vistas a sustentar sua identidade e competências profissionais, além de fortalecer sua atuação.
\end{abstract}

Palavras-chave: Terapia Ocupacional, Doenças do Sistema Nervoso Central, Atividades Cotidianas, Participação Social, Classificação Internacional da Funcionalidade, Incapacidade e Saúde.

\section{The use of activity as a tool in the process of occupational therapy intervention in neurological rehabilitation}

\begin{abstract}
This study is a theoretical consideration aiming to discuss the practice of occupational therapy in neurological rehabilitation. We focus on the use of activity as a tool for intervention processes. To this end, we propose to raise subsidies between the structure and nomenclature of the International Classification of Functioning, Disability and Health (ICF), the principles of Model of Human Occupation (MOHO), and the Motor Learning Approach for Motor Behavior Recovery. The performance of daily activities, the development of habits and their insertion on the subjects' routines can stimulate their learning and adaptation processes. This allows subjects to acquire and organize new satisfactory performance patterns for participation. In this context, the activities employed by the occupational therapist in the rehabilitation process should involve already controlled body functions, because change in motor behavior only occurs based on the modification of previously acquired skills. We emphasize the need for justification of the practice of occupational therapists in the theoretical scope of their profession, so that they can sustain their identity and professional skills, and strengthen their activities.
\end{abstract}

Keywords: Occupational Therapy, Central Nervous System Diseases, Activities of Daily Living, Social Participation, International Classification of Functioning, Disability and Health.

Autor para correspondência: Renato Nickel, Departamento de Terapia Ocupacional, Universidade Federal do Paraná, Av. Lothário Meissner, 632, B1. Didático II SD, Jd. Botânico, CEP 80210-170, Curitiba, PR, Brasil, e-mail: rnickel@ufpr.br

Recebido em 21/3/2012; Revisão em 30/7/2012; Aceito em 17/10/2012. 


\section{Introdução}

A prática dos terapeutas ocupacionais na área da reabilitação neurológica tem buscado minimizar as deficiências e incapacidades dos sujeitos pelo estímulo e desenvolvimento de habilidades necessárias para um desempenho ocupacional competente. Contudo, considera-se que, nessa área, pouco se estuda sobre a utilização da atividade como ferramenta no processo de reabilitaçáo com vistas a aumentar os níveis de atividade e participação do sujeito em seus contextos de desempenho. É possível que isso se deva a uma questáo histórica, visto que a atuação dos terapeutas ocupacionais na reabilitação física constituiu-se tradicionalmente com foco nos aspectos clínicos e indicaçôes médicas relacionadas especificamente a cada patologia, lesão ou sequela apresentada pelos pacientes (MOREIRA, 2008). Essa formação foi influenciada pelo paradigma mecanicista - predominante na época -, o que diminuiu a credibilidade do uso das ocupaçóes como ferramenta e foco de intervenção desses profissionais, devido à sua falta de cientificidade. Por volta de 1970, todavia, os terapeutas ocupacionais passaram a rediscutir sua identidade, competência e valores profissionais em busca de um novo paradigma (KIELHOFNER, 2006a).

Este artigo tem por objetivo promover uma reflexão teórica sobre o uso da atividade como ferramenta no processo de reabilitação neurológica e, consequentemente, discutir a prática do terapeuta ocupacional nesse âmbito, tendo como subsídio para argumentação a revisão de literatura e a experiência da atuação dos autores nessa área. Para isso, buscou-se orientar a perspectiva do uso da atividade por meio do Modelo Biopsicossocial da Organização Mundial da Saúde (OMS) e da nomenclatura proposta pela Classificação Internacional de Funcionalidade, Incapacidade e Saúde, em associação com o Modelo de Ocupação Humana e a Abordagem da Aprendizagem Motora para Recuperação do Comportamento Motor.

O Modelo Biopsicossocial foi proposto pela OMS em 2001, frente à dificuldade de se considerar a determinação da saúde em uma perspectiva apenas biomédica ou, por outro lado, apenas social. Nesse modelo, a atividade pode ser definida, conforme a Classificação Internacional de Funcionalidade, Incapacidade e Saúde (ORGANIZAÇĀO..., 2003, p. 243-244), como a "[...] execução de uma tarefa ou ação pelo indivíduo", no âmbito individual da funcionalidade, e está diretamente relacionada à participação do sujeito, ou seja, à perspectiva social da funcionalidade, ao "[...] envolvimento de um indivíduo em uma situação de vida real”. Esses dois aspectos são ainda qualificados de acordo com a capacidade do sujeito - seu nível máximo de funcionalidade em um ambiente ideal - e com seu desempenho, que corresponde àquilo que o indivíduo faz em seu ambiente real, englobando ainda seu envolvimento com as situaçóes de vida. Ressalta-se que, de acordo com o Modelo Biopsicossocial da OMS, a atividade e a participação são influenciadas também pelas estruturas e funçóes do corpo, por fatores ambientais e pessoais - esses do contexto -, que podem constituir-se facilitadores ou barreiras para o desempenho (ORGANIZAÇÃO..., 2003).

Já o Modelo de Ocupação Humana foi desenvolvido por Kielhofner, Burke e Heard, com vistas a articular os conceitos envolvidos em sua prática na Terapia Ocupacional, tendo suas primeiras publicaçóes no ano de 1980. De acordo com esse modelo, a ocupação humana é caracterizada pela relação dinâmica entre os subsistemas de voliçấo, habituação e capacidade de desempenho que compóem cada pessoa, sofrendo influência do ambiente no qual o sujeito está inserido, como discutido mais adiante. A volição é descrita como a motivação do sujeito para a ocupação, suas escolhas, experiências e a interpretação do seu fazer, envolvendo seu senso de efetividade no meio em que vive (causalidade pessoal), seus valores e interesses. O subsistema de habituação refere-se à capacidade de assumir comportamentos consistentes relacionados a hábitos e papéis, de acordo com uma rotina e com o ambiente em questáo. Por fim, a capacidade de desempenho é relativa à "habilidade de fazer", envolvendo componentes físicos, mentais e a subjetividade do sujeito (KIELHOFNER, 2008).

Ao contrário do Modelo Biopsicossocial, no Modelo de Ocupação Humana o conceito de atividade não é claramente definido. A atividade é apontada em um âmbito hierarquicamente menor que a ocupação, ou seja, está relacionada a uma ação ou comportamento que faz parte de um hábito ou papel, esses sim referentes à ocupação (KIELHOFNER, 2008). Assim, a ocupação humana é definida pelos autores do modelo como o

[...] desempenho das atividades de vida diária, trabalho e lazer nos contextos temporal, físico e sociocultural que caracterizam boa parte da vida humana (KIELHOFNER, 2008, p. 5)․․

A Abordagem de Aprendizagem Motora para Recuperaçáo do Comportamento Motor, por sua vez, é específica para o tratamento de pacientes com disfunçôes no sistema nervoso central. Os métodos compreendidos por essa abordagem não são pautados 
no uso da hierarquia dos reflexos para o alcance do controle dos movimentos voluntários, nem seguem as teorias tradicionais de desenvolvimento para implementação de sua intervenção (TROMBLY, 2005).

Essa abordagem requer a adoção de um modelo de prática baseada no cliente, como o Modelo de Ocupação Humana, já que as demandas devem ser identificadas de acordo com as características e interesses de cada paciente. Assim, as atividades empregadas na intervenção devem ser significativas para o sujeito, coerentes com seus interesses e com os papéis desempenhados por ele. $\mathrm{O}$ terapeuta ocupacional realiza, entáo, análise das atividades nas quais o sujeito apresenta demandas, avalia seus padrôes de movimento e considera a influência de fatores pessoais e ambientais no desempenho do sujeito. Nessa abordagem, o desempenho das próprias atividades é empregado na intervenção em associação a outros métodos de recuperação das funçôes do corpo, quando possível. Em suma, pode-se dizer que essa abordagem tem foco no paciente, na ocupaçáo e na prática como forma de alcançar a aprendizagem motora, além de admitir a influência de fatores pessoais e ambientais nesse desempenho (BASS-HAUGEN; MATHIOWETZ; FLINN, 2005).

Ressalta-se ainda que o uso de atividades intencionais e a análise de atividades são habilidades essenciais dos terapeutas ocupacionais, descritas entre as "ferramentas legítimas" da Terapia Ocupacional propostas por Mosey (1986, apud HAGEDORN, 1999) e as "ferramentas da prática" citadas por Willard e Spackman (1992, apud HAGEDORN, 1999). Portanto, o termo "ferramenta" foi empregado para categorizar a atividade no processo de reabilitação neurológica no presente artigo.

Sob a perspectiva assumida nesse trabalho em relação à atividade não apenas como meio, recurso de intervenção, mas especialmente como fim do processo de reabilitação, como ação que compóe e caracteriza uma ocupação, pode-se definir Terapia Ocupacional como descrita pela Federação Mundial de Terapeutas Ocupacionais:

Terapia Ocupacional é uma disciplina da saúde que diz respeito a pessoas com deficiência, déficit ou incapacidade física ou mental, temporária ou permanente. O Terapeuta Ocupacional profissionalmente qualificado envolve o paciente em atividades destinadas a promover o restabelecimento e o máximo uso de suas funçôes, com o propósito de ajudá-lo a fazer frente às demandas de seu ambiente de trabalho, social, pessoal e doméstico e a participar da vida em seu mais pleno sentido. (WORLD..., 2001, p. 12).

\section{Argumentação teórica}

$\mathrm{Na}$ perspectiva de Kielhofner (2005), o desenvolvimento da Classificação Internacional de Funcionalidade, Incapacidade e Saúde e o uso de sua nomenclatura e estrutura pelos terapeutas ocupacionais permite enfatizar o desempenho nas atividades para alcance da participaçáo como objetivo final das intervençóes de Terapia Ocupacional, ou seja, auxiliar e estimular o sujeito no desempenho de suas atividades em diferentes contextos. Como já apontado, o Modelo de Ocupação Humana enfoca o fazer e, bem como a CIF, admite a interação desse desempenho na atividade e participação com fatores pessoais e ambientais.

Especificamente em relação ao Modelo de Ocupação Humana, alterações nos subsistemas de volição, habituaçáo e capacidade de desempenho provocam alteraçóes no pensar, sentir e fazer do sujeito em relação a suas ocupações - o que ocorre também de forma recíproca. Desse modo, quando os padrôes de desempenho náo são mais adequados para o indivíduo, ele muda sua forma de pensar, sentir e fazer acerca de suas ocupaçóes e assume novos padróes de volição, habituação e capacidade de desempenho. Tais padrōes são repetidos no ambiente no qual está inserido até que um novo padrão de desempenho satisfatório para o sujeito torne-se hábito e seja integrado a uma rotina (KIELHOFNER, 2008).

Tais conceitos envolvidos no Modelo de Ocupação Humana podem ser considerados também muito próximos à perspectiva da aprendizagem, uma vez que o desempenho de ocupaçóes pelo sujeito em uma rotina implica na relação dinâmica entre os postulados descritos, levando-o a assumir hábitos. Os hábitos podem ser definidos como tendências adquiridas a partir dos padróes adotados para o desempenho das ocupaçóes e modos de responder a demandas, desenvolvidos através da repetição de ações, pensamentos e sentimentos, tornando-se automáticos e consistentes em situaçôes e contextos ambientais familiares. Hábitos, se envolvidos na rotina podem, portanto, determinar e organizar os padróes empregados e o uso de funçóes corporais específicas para o desempenho do sujeito nas ocupaçóes, em seus diversos contextos (KIELHOFNER, 2008).

Dessa forma, em situaçóes nas quais a capacidade para o desempenho encontra-se diminuída, como no caso do sujeito com uma condição neurológica, ele pode ter alguns de seus hábitos prejudicados, fazendo com que novos hábitos sejam assumidos em sua vida cotidiana. Ou seja, quando os hábitos do sujeito deixam de regular automaticamente seu fazer, "[...] atenção e esforço adicionais são necessários, removendo a 
facilidade e a eficiência que geralmente acompanham as rotinas diárias" (KIELHOFNER, 2008, p. 58)².

A aprendizagem motora está justamente relacionada à capacidade do homem de se adaptar a novas situaçôes de vida e de modificar seu ambiente e ocorre a partir de um processo constante de "estabilidade-instabilidade-estabilidade". Sob essa perspectiva, frente a novas demandas de habilidades para o desempenho de atividades, o sujeito passa por um processo adaptativo que pode ser compreendido em duas fases: estabilização e adaptação. A primeira refere-se à padronizaçáo dos movimentos pelo sujeito a partir de seus erros, ou seja, a partir de feedback negativo. Já a fase de adaptação é relativa à flexibilizaçấo e à adequação dos movimentos àquelas demandas impostas pelas atividades. Essa fase ocorre sempre em um nível inferior ao da estabilização, ou seja, a modificação do comportamento motor só é possível se baseada em habilidades já adquiridas e controladas pelo sujeito. Tal processo resulta na automatização dos movimentos, característica ao final do processo de aprendizagem motora (TANI, 2000; BARROS, 2006).

Particularmente em relação ao uso da atividade no processo de reabilitaçáo do sujeito com uma condição neurológica, o terapeuta ocupacional, em sua especificidade, pode atuar sob o escopo da Abordagem de Aprendizagem Motora para Recuperação do Comportamento Motor, com o objetivo de maximizar o desempenho ocupacional desse sujeito por meio do desempenho de suas próprias atividades cotidianas (TROMBLY, 2005).

Acredita-se que o controle dos movimentos voluntários pode ser retomado no desempenho dessas atividades, estimulando habilidades de desempenho e admitindo alteraçóes ambientais para, no caso da intervenção do terapeuta ocupacional, retomar o máximo desempenho ocupacional do sujeito após a lesão cerebral (TROMBLY, 2005; WOODSON, 2005).

Enfatiza-se que estimular o desempenho de atividades pelo paciente é importante também para manter os processos plásticos do sistema nervoso central. A prática da Aprendizagem Motora para Recuperaçáo do Comportamento Motor, por sua vez, permite que as funções neuromusculoesqueléticas e relacionadas ao movimento sejam aperfeiçoadas, sendo que a natureza do treinamento, ou seja, sua aplicação prática no cotidiano do sujeito é essencial para que a função motora seja estimulada de modo mais eficaz (UMPHRED et al., 2009).

Ressalta-se ainda a importância da intensidade do treinamento, devendo-se salientar o resultado esperado nele. A expectativa do sujeito mostra-se fundamental na melhora da funcionalidade, uma vez que um paciente realmente engajado na terapia apresenta maiores chances de alcançar um desempenho satisfatório. Aponta-se também a importância de proporcionar feedback ao paciente, para que ele observe os resultados do treino em seu comportamento motor (GAUTHIER et al., 2008).

Para essas intervençôes, sugere-se que as atividades envolvidas no treino tenham sua dificuldade graduada conforme a melhora na funcionalidade do paciente, constituindo um desafio com possibilidades de sucesso. Tais atividades devem estar voltadas a um objetivo com execução repetida, já que a prática levará ao aprendizado motor. É importante promover estímulos sensoriais de força adequada, para que seja possível provocar movimentos de padrão normal e evitar movimentos anormais, em atividades significativas e adequadas aos interesses do paciente. Deve-se buscar também a aplicação das funções neuromusculoesqueléticas e relacionadas ao movimento em atividades realizadas nos diferentes contextos de desempenho do paciente (UMPHRED et al., 2009).

Nota-se, dessa forma, que a utilização dos modelos baseados na Classificaçáo Internacional de Funcionalidade, Incapacidade e Saúde, o Modelo de Ocupação Humana e a Abordagem de Aprendizagem Motora para Recuperação do Comportamento Motor permitem pensar na reabilitação do sujeito com condição neurológica com práticas voltadas à atividade e à participação desse sujeito, envolvendo a recuperação de suas funções neuromusculoesqueléticas e relacionadas ao movimento e a aplicação dessas em atividades significativas.

Entretanto, as funções do corpo que o sujeito apresenta e controla devem ser estimuladas e empregadas nas atividades sem necessariamente constituir um desafio, possibilitando que o sujeito adeque suas funções neuromusculoesqueléticas e relacionadas ao movimento, o uso da mão e do braço e o uso fino da mão nas atividades, permitindo sua habituação, inclusão e prática na rotina diária. $\mathrm{Ou}$ seja, a repetição das funçôes do corpo controladas pelo sujeito na atividade leva a padrôes de desempenho organizados, os quais podem constituir hábitos e são incluídos na rotina do sujeito, estimulando a aprendizagem motora e favorecendo a melhora permanente em sua funcionalidade.

A fim de facilitar a compreensão da relação proposta entre os conceitos do Modelo de Ocupação Humana, da Abordagem de Aprendizagem Motora para Recuperação do Comportamento Motor e a nomenclatura fornecida pela Classificação Internacional de Funcionalidade, optou-se pela apresentação em Quadro 1. Para a confecçáo dela, tomou-se como base a comparaçáo já feita por Kramer, Bowyer e Kielhofner (2008) entre conceitos do Modelo de Ocupação Humana e da Classificação Internacional de Funcionalidade, Incapacidade e Saúde. 
Quadro 1. Quadro comparativo de termos: Modelo de Ocupação Humana, Abordagem de Aprendizagem Motora para Recuperação do Comportamento Motor e Classificação Internacional de Funcionalidade, Incapacidade e Saúde.

\begin{tabular}{|c|c|c|}
\hline Modelo de ocupação humana & $\begin{array}{c}\text { Abordagem de aprendizagem } \\
\text { motora para recuperação do } \\
\text { comportamento motor }\end{array}$ & $\begin{array}{c}\text { Classificação internacional } \\
\text { de funcionalidade, } \\
\text { incapacidade e saúde }\end{array}$ \\
\hline Participação ocupacional & - & Participação \\
\hline Desempenho ocupacional & Desempenho de tarefas funcionais & Atividade \\
\hline Padrões de desempenho & Repetição/aprendizagem & $\begin{array}{c}\text { Atividade/participação/fatores } \\
\text { ambientais }\end{array}$ \\
\hline Rotina & Repetição/aprendizagem & Atividade/participação \\
\hline Volição & Motivação & $\begin{array}{l}\text { Funções do corpo: Funções } \\
\text { mentais }\end{array}$ \\
\hline Habituação & Repetição/aprendizagem & $\begin{array}{c}\text { Funções do corpo: Funções } \\
\text { mentais }\end{array}$ \\
\hline Capacidade de desempenho & Capacidade funcional & Funções do corpo/capacidade \\
\hline $\begin{array}{l}\text { Ambiente físico, social, cultural, } \\
\text { condições políticas e econômicas }\end{array}$ & Ambiente & Fatores ambientais \\
\hline Habilidades motoras & Controle motor & $\begin{array}{c}\text { Funções do corpo: Funções } \\
\text { neuromusculoesqueléticas e } \\
\text { relacionadas ao movimento - } \\
\text { Funções de controle voluntário dos } \\
\text { movimentos } \\
\end{array}$ \\
\hline Habilidades de processo & Aprendizagem & $\begin{array}{l}\text { Funções do corpo: Funções } \\
\text { mentais }\end{array}$ \\
\hline $\begin{array}{l}\text { Habilidades de comunicação/ } \\
\text { interação }\end{array}$ & Atenção & $\begin{array}{l}\text { Funções do corpo: Funções } \\
\text { mentais }\end{array}$ \\
\hline
\end{tabular}

Fonte: autores.

\section{Conclusão}

Embora as definições de atividade sejam variáveis entre os modelos apresentados, pode-se afirmar que o termo "atividade" assume nos três modelos uma perspectiva muito semelhante em relação à ação, àquilo que um sujeito faz e que está compreendido em um aspecto social mais amplo. Da mesma forma, "ocupação" e "participação" são denominaçôes muito próximas nesses modelos, ao designar o âmbito social do fazer, o papel do sujeito no contexto onde está inserido, que, de certa forma, determina as atividades que ele desempenha no cotidiano. Não somente na nomenclatura, observa-se uma consonância entre os modelos ao assumirem a influência de fatores pessoais e ambientais na atividade e participação do sujeito. Além disso, tanto o Modelo de Ocupação Humana quanto a Abordagem de Aprendizagem Motora para Recuperaçáo do Comportamento Motor apresentam foco no significado das atividades para o sujeito e na prática, no processo de habituação para uma aprendizagem plena.

$\mathrm{Na}$ prática clínica, de acordo com o exposto, acreditamos que em se tratando de reabilitação neurológica a autonomia no desempenho de atividades cotidianas seja buscada na intervençáo do terapeuta ocupacional com vistas à retomada da participação do sujeito.

Não há como desconsiderar a natureza ocupacional do ser humano. As ocupações nas quais o homem é motivado a se engajar influenciam e organizam seu comportamento, além de conferirem significado à sua vida (KIELHOFNER, 2006b).

Admite-se dessa forma que a motivação é o que leva o sujeito a se engajar também no processo de reabilitação. A atividade é, portanto, ferramenta singular, própria do terapeuta ocupacional, a qual permite ao sujeito (paciente) e dá ao terapeuta todos os elementos necessários para aplicar uma Abordagem de Aprendizagem Motora para Recuperação do Comportamento Motor. Atividades possíveis de serem desempenhadas podem tornar-se rotina e constituir ganhos permanentes, permitindo aos sujeitos o envolvimento e situaçóes de vida real.

Assim, se a atividade, enquanto ferramenta, faz parte tanto da competência quanto da identidade profissional dos terapeutas ocupacionais (KIELHOFNER, 2006c), cabe a eles retomarem 
as discussóes iniciadas nas décadas de 1960 e 1970, após a segunda crise paradigmática da Terapia Ocupacional.

Faz-se necessário, então, que os terapeutas ocupacionais fundamentem sua prática no escopo teórico próprio da profissão em relação à ocupação como aspecto central, de modo a garantir sua especificidade e fortalecer sua atuaçáo.

\section{Referências}

BARROS, J. A. C. Estrutura de prática e processo adaptativo em aprendizagem motora: efeitos da especificidade da tarefa. 2006. 99 f. Dissertação (Mestrado em Biodinâmica do Movimento Humano)-Escola de Educação Física e Esporte, Universidade de São Paulo, São Paulo, 2006. Disponível em: <http://www.teses.usp.br/teses/ disponiveis/39/39132/tde-11082008-085547/>. Acesso em: 23 fev. 2012.

BASS-HAUGEN, J.; MATHIOWETZ, V.; FLINN, N. Abordagem de Terapia Ocupacional Orientada à Tarefa para Otimizar o Comportamento Motor. In: TROMBLY, C. A.; RADOMSKI, M. V. Terapia Ocupacional para Disfunções Físicas. 5. ed. São Paulo: Santos, 2005. p. 481-499.

HAGEDORN, R. Habilidades e processos essenciais. In: HAGEDORN, R. Fundamentos da Prática em Terapia Ocupacional. São Paulo: Dynamis Editorial, 1999. p. 29-48.

GAUTHIER, L. V. et al. Remodeling the Brain: plastic structural brain changes produced by different motor therapies after stroke. Stroke, Dallas, v. 39, n. 5, p. 15201525, 2008. PMid:18323492 PMCid:PMC2574634. http://dx.doi.org/10.1161/STROKEAHA.107.502229

KIELHOFNER, G. Rethinking Disability and What To Do About It: disability studies and its implications for occupational therapy. American Journal of Occupational Therapy, Bethesda, v. 59, n. 5, p. 487-496, Sept/Oct 2005. http://dx.doi.org/10.5014/ajot.59.5.487

KIELHOFNER, G. El desarrollo del conocimiento de la terapia ocupacional. In: KIELHOFNER, G. Fundamentos conceptuales de la Terapia Ocupacional. 3. ed. Buenos Aires: Médica Panamericana, 2006a. p. 27-63.

KIELHOFNER, G. El paradigma contemporáneo: un retorno a la ocupación como el centro de la profesión. In: KIELHOFNER, G. Fundamentos conceptuales de la Terapia Ocupacional. 3. ed. Buenos Aires: Médica Panamericana, 2006b. p. 64-71.

KIELHOFNER, G. Introducción. In: KIELHOFNER, G. Fundamentos conceptuales de la Terapia Ocupacional. 3. ed. Buenos Aires: Médica Panamericana, 2006c. p. 2-9.

KIELHOFNER, G. Model of Human Occupation: theory and application. 4. ed. Baltimore: Lippincott Williams \& Wilkins, 2008.

KRAMER, J.; BOWYER, P.; KIELHOFNER, G. The Model of Human Occupation, the ICF, and the Occupational Therapy Practice Framework: connections to support best practice around the world. In: KIELHOFNER, G. Model of Human Occupation: theory and application. 4. ed. Baltimore: Lippincott Williams \& Wilkins, 2008. p. 519-531.

MOREIRA, A. B. Terapia Ocupacional: história crítica e abordagens territoriais/comunitárias. Vita et Sanitas, Trindade, v. 2, n. 2, p. 79-91, 2008. Disponível em: <http://www.fug.edu.br/revista_2/pdf/artigo_to.pdf>. Acesso em: 20 set. 2013.

ORGANIZAÇÃO MUNDIAL DA SAÚDE - OMS. CIF: classificação internacional de funcionalidade, incapacidade e saúde. São Paulo: Edusp, 2003.

TANI, G. Processo adaptativo em aprendizagem motora: o papel da variabilidade. Revista Paulista de Educação Física, São Paulo, p. 55-61, 2000. Suplemento 3. Disponível em: <http://citrus.uspnet.usp.br/eef/uploads/arquivo/ v14\%20supl3\%20artigo6.pdf $>$. Acesso em: 20 fev. 2012.

TROMBLY, C. A. Fundamentos Conceituais para a Prática. In: TROMBLY, C. A.; RADOMSKI, M. V. Terapia Ocupacional para Disfunçóes Físicas. 5. ed. São Paulo: Santos, 2005. p. 1-15.

UMPHRED, D. A. et al. Intervenções em Pacientes com Limitaçôes do Movimento. In: UMPHRED, D. A. (Org.). Reabilitação Neurológica. 5. ed. Rio de Janeiro: Elsevier, 2009. p. 153-234.

WORLD FEDERATION OF OCCUPATIONAL THERAPISTS - WFOT. Definição e Função da Terapia Ocupacional da WFOT. Definiçóes de Terapia Ocupacional. 1993. Disponível em: <http://www. salesianolins.br/areaacademica/materiais/posgraduacao/ Livro_TO/DefinicoesTO.pdf>. Acesso em: 03 maio 2011. WOODSON, A. M. Acidente Vascular Cerebral. In: TROMBLY, C. A.; RADOMSKI, M. V. Terapia Ocupacional para Disfunções Físicas. 5. ed. São Paulo: Santos, 2005. p. 817-853.

\section{Contribuição dos Autores}

Diane Priscila Stoffel: responsável pela elaboração do texto. Renato Nickel: responsável pelo auxílio na elaboração do texto e revisão final.

\section{Notas}

1 Traduzido pelos autores.

${ }^{2}$ Traduzido pelos autores. 